\title{
Heterociclos: pequeñas y maravillosas estructuras en el organismo humano
}

\author{
Delia Hernández Romero, Oscar García Barradas, \\ Raúl Colorado Peralta y Esmeralda Sánchez Pavón
}

\section{Resumen}

La capacidad de un ser vivo para realizar algunas funciones depende de una serie de compuestos químicos en los cuales en muchas ocasiones están implicados pequeños anillos llamados heterociclos. Estos a su vez pueden formar parte de diferentes estructuras capaces de darnos características físicas como el ADN, el cual tiene toda la información genética de nuestros padres; los aminoácidos, encargados de realizar funciones específicas en nuestro organismo; las hormonas, implicadas en algunos momentos de felicidad y placer a lo largo de nuestra vida; entre otras estructuras. Por lo anterior, es importante reconocer a los heterociclos, ya que son parte esencial de los seres vivos.

Palabras clave: heterociclos, bases púricas, bases pirimidínicas, vitaminas, grupo hemo, aminoácidos, hormonas.

\section{HETEROCYCLES: SMALL AND WONDERFUL STRUCTURES IN THE HUMAN ORGANISM}

\begin{abstract}
The ability of a living organism to perform certain functions depends on a series of chemical compounds, in which small rings called heterocycles are often involved. These, in turn, can be part of different structures capable of giving us physical characteristics such as DNA, which has all the genetic information of our parents; aminoacids, that are responsible for carrying out specific functions in our body; hormones, involved in some moments of happiness and pleasure throughout our lives; among other structures. Therefore, it is important to recognize these small heterocyclic structures with which our body stays in contact throughout life.
\end{abstract}

Key words: heterocycles, purine bases, pyrimidine bases, vitamins, heme, amino acids, hormones. 
"Heterociclos: pequeñas y maravillosas estructuras en el organismo humano" Delia Hernández Romero, Oscar García Barradas, Raúl Colorado Peralta y Esmeralda Sánchez Pavón Vol. 19, Núm. 4, julio-agosto 2018 Revista Digital Universitaria

\section{Delia Hernández Romero}

deliahernandez@uv.mx

Profesora de tiempo completo en la Facultad de Ciencias Químicas de la Universidad Veracruzana, campus Orizaba. Estudió la licenciatura de Químico Farmacéutico Biólogo en la misma universidad. Realizó sus estudios de doctorado en la Universidad de Barcelona. Una vez finalizada la tesis de doctorado, el siguiente paso fue incorporarse a desarrollar proyectos I+D de empresas farmacéuticas en la Unidad de Química Combinatoria del Parque Científico de Barcelona, donde permaneció trabajando durante cuatro años. En el año 2012 se incorporó a la Universidad Veracruzana con beca de repatriación del CONACYT y en el 2013 obtuvo su plaza en la Universidad Veracruzana. Actualmente pertenece al sistema nacional de investigadores del CONACYT. La investigación que realiza se basa en la síntesis de derivados de heterociclos con actividad biológica, así como en el aislamiento de metabolitos secundarios de plantas medicinales.

\section{Oscar García Barradas}

osgarcia@uv.mx Investigador titular de la Unidad de Servicios de Apoyo en Resolución Analítica (SARA), profesor titular del posgrado en Ciencias Alimentarias del Instituto de Ciencias Básicas y profesor titular de la Facultad de Química Farmacéutica Biológica de la Universidad Veracruzana, campus Xalapa. Responsable del Laboratorio de Resonancia Magnética Nuclear de la Unidad SARA. Actualmente es investigador nacional nivel I, otorgado por el Sistema Nacional de Investigadores del CONACYT.

Es egresado de la Facultad de Química Farmacéutica Biológica de la Universidad Veracruzana. Obtuvo el grado de Doctor en Ciencias por el Departamento de Química del Centro de Investigación y de Estudios Avanzados del Instituto Politécnico Nacional (CINVESTAV). Sus intereses en investigación se centran en la síntesis de derivados con actividad biológica potencial, en la estereoquímica y la elucidación estructural mediante técnicas espectroscópicas.

\section{Raúl Colorado Peralta}

racolorado@uv.mx

Profesor de tiempo completo en la Facultad de Ciencias Químicas de la Universidad Veracruzana, campus Orizaba. Realizó sus estudios de licenciatura en la misma universidad y el Doctorado en Ciencias Químicas en el Departamento de Química del CINVESTAV, IPN; el posdoctorado en el Departamento de Química Inorgánica y Nuclear de la Facultad de Química de la UNAM. Durante su trayectoria académica realizó estancias de Investigación en la Universidad de Tecnología de Viena en Austria y en la Universidad de Wisconsin-Madison en Estados Unidos. Actualmente es investigador nacional nivel I, otorgado por el Sistema Nacional de Investigadores del CONACYT. Sus intereses de investigación se centran en la síntesis de nuevos derivados luminiscentes de bencimidazol.

\section{Esmeralda Sánchez Pavón}

esmesanchez@uv.mx

Profesor de tiempo completo en la Facultad de Ciencias Químicas de la Universidad Veracruzana, campus Orizaba. Es egresada de la Facultad de Ciencias Químicas de la Universidad Veracruzana de la carrera de Químico Industrial, tiene estudios de maestría en Ciencias en Química Bioorgánica por la Escuela Nacional de Ciencias Biológicas del IPN. Obtuvo el grado de Doctora en Química en el 2004 en la Universidad de Alcalá en España. Sus intereses de investigación se basan en la síntesis de derivados de nitroimidazol con actividad antiparasitaria y antibacterial. 


\section{Introducción}

Los compuestos orgánicos están formados por carbono e hidrógeno; pueden ser lineales o bien en forma de ciclo y tienen una gran diversidad de estructuras que van desde los compuestos con un solo átomo de carbono, como es el caso del metano $\left(\mathrm{CH}_{4}\right)$, hasta aquellos compuestos que tienen una cantidad indefinida de carbonos en su estructura, como por ejemplo las proteínas. Una gran parte de estos compuestos orgánicos son los heterociclos, los cuales se diferencian por la presencia, además del carbono e hidrógeno, de nitrógeno, azufre, oxígeno, entre otros heteroátomos, y por formar parte de un anillo; lo cual les proporciona características especiales para estar presentes como estructuras químicas primordiales en los seres vivos.

Al hablar de algunas estructuras y saber cuál es su función, podremos identificar de mejor manera los heterociclos de los que se forman. Algunos ejemplos de estos compuestos químicos de gran relevancia se mencionan a continuación.

\section{Bases púricas y pirimidínicas}

Para que las características de un ser humano se transmitan a sus descendientes se requiere la participación del ácido desoxirribonucleico (ADN) ya que éste contiene la información genética en el núcleo de la célula. Por su parte, el ácido ribonucleico (ARN) es su contacto con el exterior del núcleo para que toda esa información salga y se pueda expresar en cada ser vivo. Su estructura parece muy complicada; sin embargo, si identificamos de manera específica alguna de las partes que los componen podremos visualizarlos de manera más clara. Estas grandes diseñadoras del organismo se pueden distinguir entre sí por diferencias entre

Imagen 1. cada uno de los heterociclos que contienen. La adenina, timina, guanina y citosina se encuentran en el ADN, además de una estructura adicional que se llama desoxirribosa; mientras que el aRN en lugar de timina contiene uracilo y

DNA

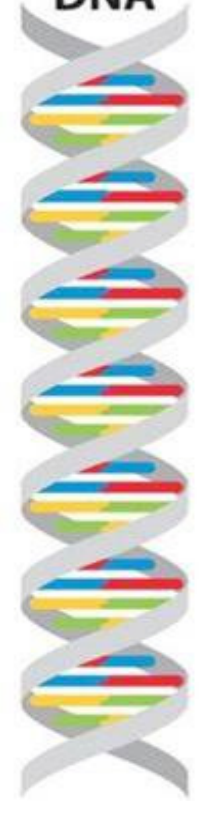

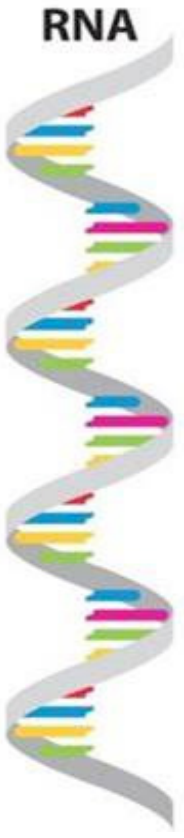
ribosa (Mathews et al., 2002).

La adenina y guanina son también llamadas bases púricas (ver figura 1), están formadas por la fusión de dos heterociclos uno de seis miembros y uno de cinco, en los cuales, además de contener carbono e hidrógeno, también 
Figura 1.

Bases púricas.

Figura 2.

Bases pirimídicas.

Figura 3.

Pentosas.

contienen nitrógeno como parte del ciclo. A este heterociclo en particular se le llama purina.

Heterociclo Purina

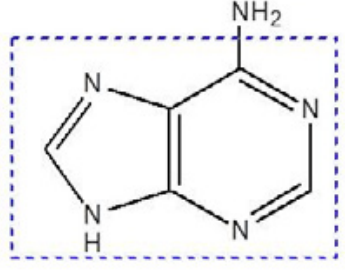

ADENINA

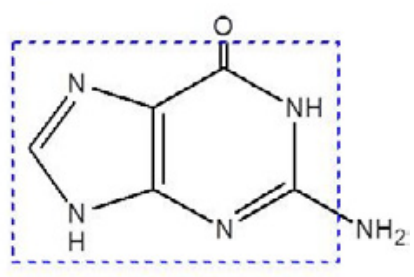

GUANINA

Por su parte, la citosina, uracilo y timina (figura 2) son llamadas bases pirimídicas y su característica es que son un anillo de seis miembros con dos átomos de nitrógeno llamado pirimidina.

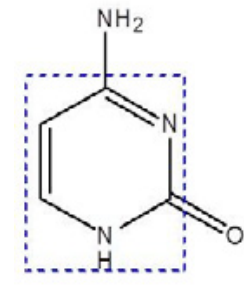

CITOSINA
Heterociclo Pirimidina

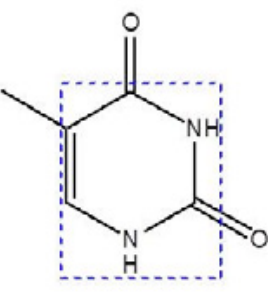

TIMINA

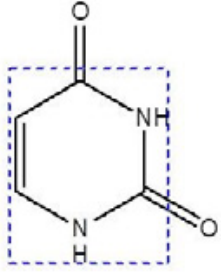

URACILO
Por su parte, la ribosa presente en el ARN y la desoxirribosa presente en el ADN son heterociclos derivados del furano (figura 3).

Heterociclo tetrahidrofurano
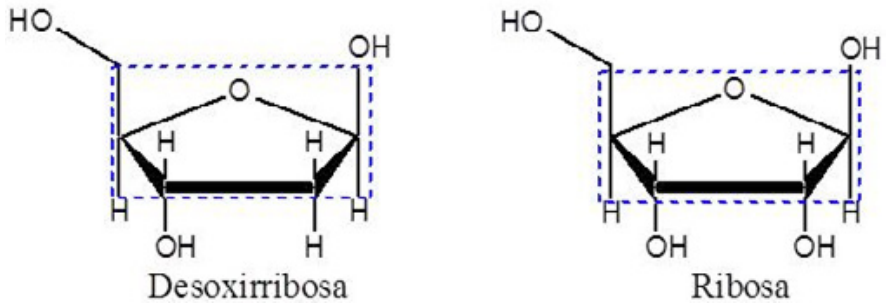

Como ya se mencionó, los ácidos nucleicos (ADN y ARN) tienen la gran responsabilidad de almacenar y transmitir la información genética. Para llevar a cabo esta actividad generan aminoácidos los cuales son la base estructural para formar proteínas. 
Imagen 2.

Proteína.

Figura 4.

Heterociclos en los aminoácidos.

\section{Aminoácidos}

Los aminoácidos son compuestos químicos que, como su nombre lo indica, contienen los grupos funcionales amina y ácido carboxílico. Estos compuestos funcionan como bloques de construcción unidos secuencialmente para formar proteínas (Mathews et al., 2002).

De los aminoácidos que se encuentran en las proteínas, tres de ellos, la his-

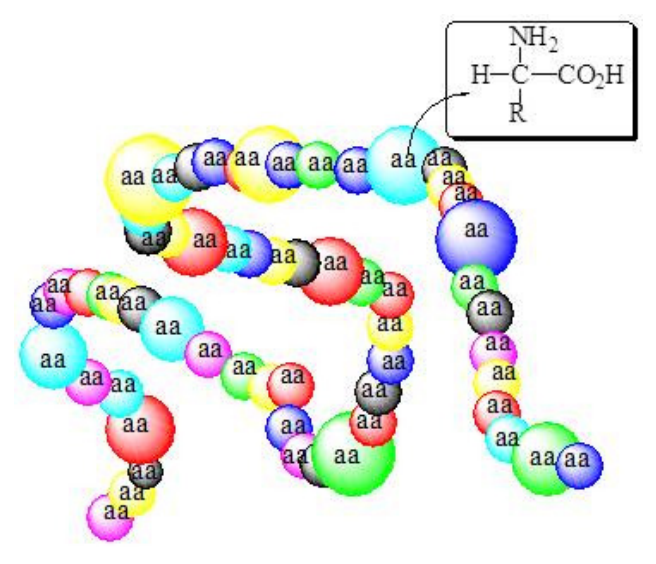
tidina, la prolina y el triptófano son compuestos heterocíclicos (Figura 4). Estos aminoácidos además de formar parte de las proteínas tienen funciones específicas en el organismo.
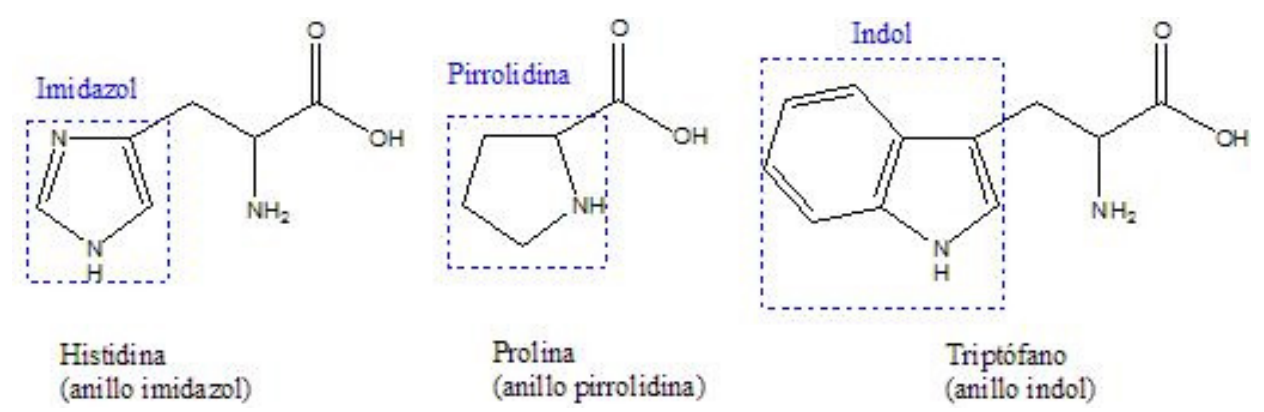

En la histidina se encuentra el imidazol, un heterociclo aromático de cinco miembros que incorpora dos nitrógenos. Este aminoácido es imprescindible en la producción de glóbulos rojos y blancos en la sangre (de ahí que la encontremos de forma abundante en la hemoglobina); además de ayudar a reducir la presión arterial, es útil en la eliminación de metales pesados y protege al organismo de los daños por radiación.

En el caso de la prolina, el heterociclo que incorpora es llamado pirrolidina, este aminoácido tiene una función en la formación de colágeno, el cual, entre otras funciones, es el encargado de mantener la elasticidad en nuestra piel para vernos más jóvenes.

El triptófano tiene un heterociclo llamado indol, este aminoácido tiene un efecto calmante en el sistema nervioso, por lo cual ayuda a calmar la ansiedad y la depresión, y además alivia el insomnio al inducir el sueño. 


\section{Vitaminas}

Las vitaminas cumplen una función muy importante en nuestro cuerpo ya que intervienen en diversas etapas del metabolismo (Murray et al., 2010; Pardo, 2004). Algunas de las vitaminas que son esenciales para el buen desarrollo y crecimiento de nuestro cuerpo y que tienen incorporado un heterociclo en su estructura se mencionan a continuación.

\section{Tiamina o vitamina B1}

La vitamina B1 tiene dos heterociclos en su estructura, el

Figura 5. anillo de tiazol, un heterociclo de cinco miembros que incorpora nitrógeno y azufre; y la pirimidina, un anillo de seis

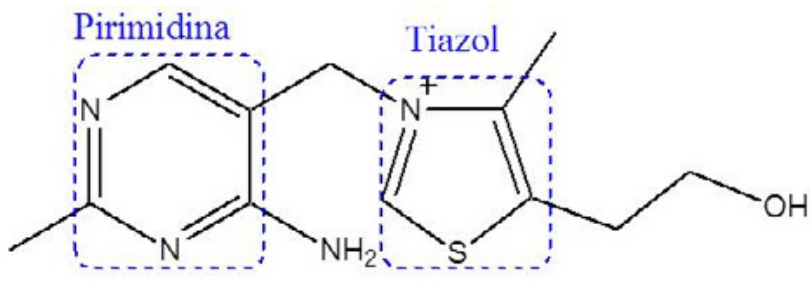
miembros con dos átomos de nitrógeno en su estructura (figura 5). Una de sus funciones es la síntesis de hidratos de carbono, lo cual nos permite tener energía.

Figura 6. Riboflavina o vitamina B2.

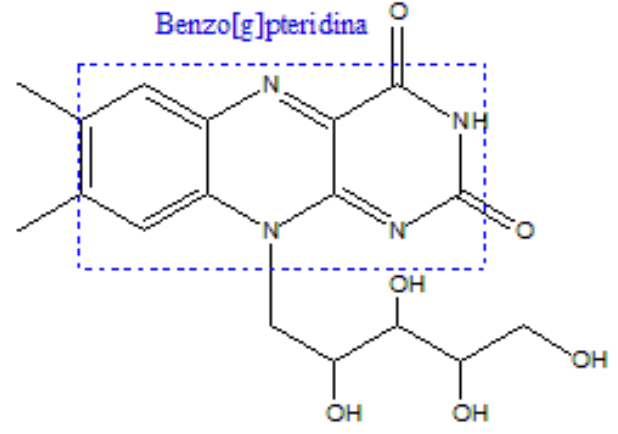

Riboflavina o vitamina $B 2$

\section{Riboflavina o vitamina B2}

La vitamina B2 tiene un heterociclo fusionado de tres sistemas aromáticos, a través de enlaces carbono-carbono llamado benzo[g] pteridina (figura 6). Es importante para el crecimiento corporal y la producción de glóbulos rojos y ayuda en el metabolismo de los carbohidratos, grasas y especialmente en el metabolismo de las proteínas.

\section{Vitamina B3}

La vitamina B3 tiene un heterociclo de seis miembros con un heteroátomo de nitrógeno y es llamado piridina (figura 7). Esta vitamina interviene en la

Figura 7. respiración celular. También se encarga de la resVitamina B3. tauración de ADN, permite la producción de neurotransmisores, la síntesis de hormonas, y colabora en el funcionamiento sano y completo del sistema nervioso.

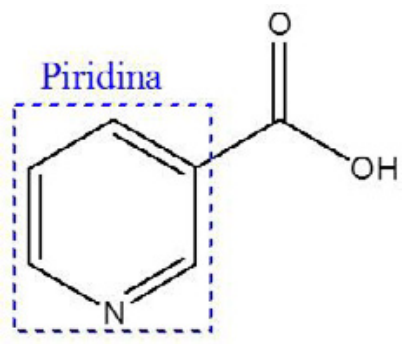


Figura 8. (figura 8). Interviene en el metabolismo de los Piridoxina o vitamina B6.

\section{Piridoxina o vitamina B6}

La vitamina B6 también tiene un heterociclo de piridina como base principal de su estructura carbohidratos, las grasas y las proteínas, pero su función principal es la asimilación de los aminoácidos. Actúa también en la degradación del colesterol y en la formación de anticuerpos.

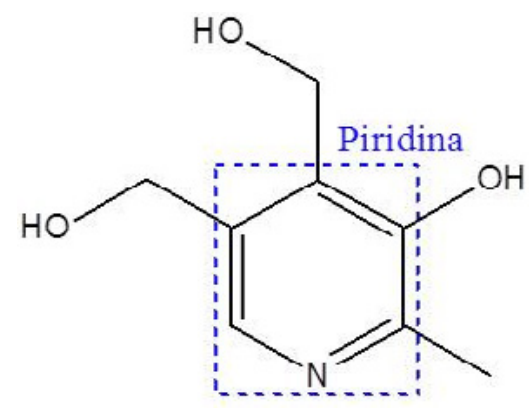

Figura 9.

Cobalamina o vitamina B12.

\section{Cobalamina o vitamina B12}

Es una macromolécula que tiene como parte de su estructura cuatro heterociclos diferentes $\mathrm{A}=3,4$-dihidro-2 $\mathrm{H}$-pirrol, $\mathrm{B}=$ pirrolidina, $\mathrm{C}=$ bencimidazol, $\mathrm{D}=$ tetrahidrofurano, como se muestra en la figura 9. Es esencial para el funcionamiento en general de todas las células y en específico para la formación de los glóbulos rojos en la médula de los huesos, interviene en los procesos del sistema nervioso y en el tracto gastrointestinal.

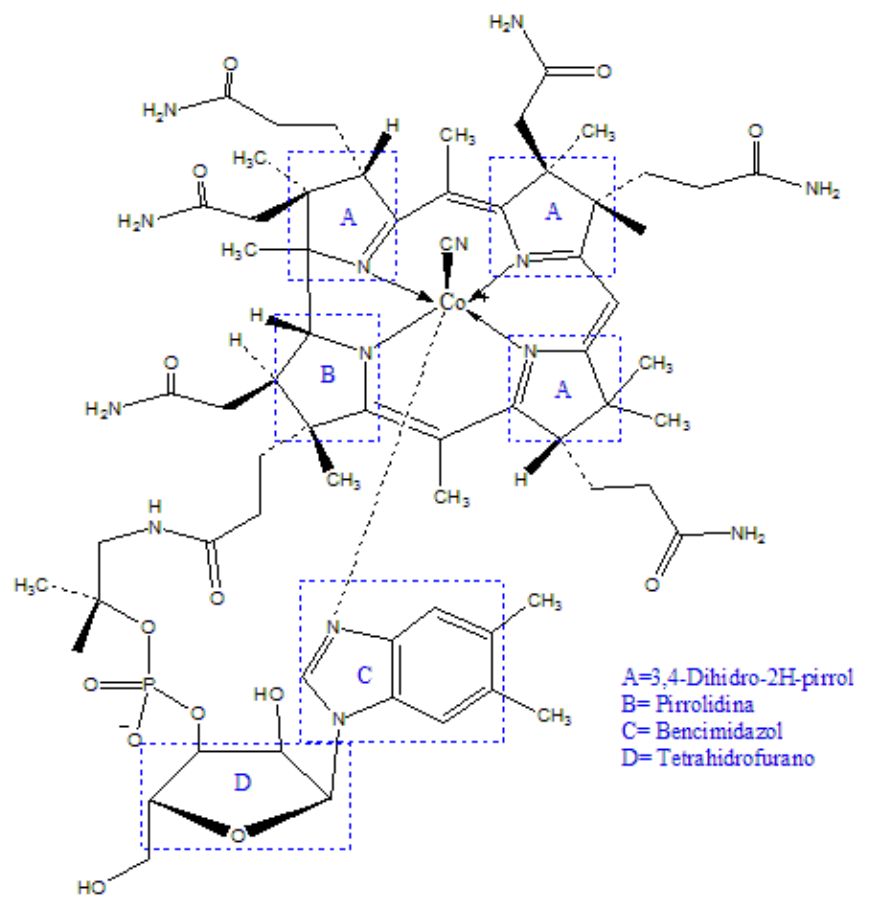

\section{Vitamina B9}

Su nombre deriva del latín folium que significa hoja, porque fue aislado por primera vez de las hojas de espinaca, por lo cual también se le llama ácido fólico o folacina. El heterociclo es una fusión de dos sistemas aromáticos a través de 
Figura 10.

Vitamina B9 o ácido fólico.
Figura 11. Vitamina $\mathrm{H}$.
Figura 12. Vitamina $C$. un enlace carbono-carbono y su nombre común es pteridina (figura 10). Es necesaria para la formación de proteínas, eritrocitos y leucocitos, además participa en el metabolismo de carbohidratos y ácidos grasos. Durante el embarazo es indispensable para el correcto funcionamiento del organismo de la mujer y la correcta formación del bebé.

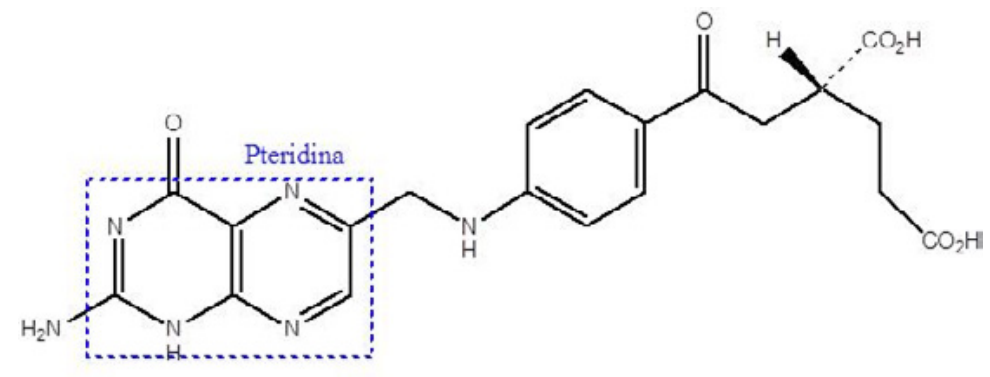

\section{Vitamina $\mathbf{H}$}

La vitamina H está compuesta de un anillo ureico (imidazoleínico) fusionado con un anillo de tetrahidrotiofeno (figura 11). Participa en la formación de ácidos grasos y en la liberación de energía procedente de los carbohidratos.

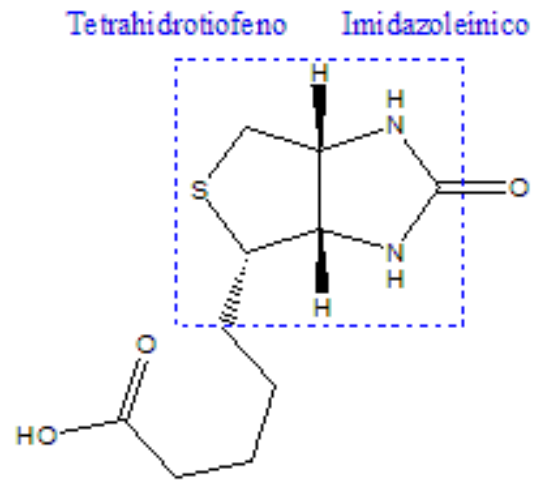

\section{Vitamina C}

La vitamina C tiene incorporado el heterociclo 2,5-dihidrofurano (figura 12). Es importante en la formación y conservación del colágeno, y en la formación de huesos y dientes. Interviene en el metabolismo de las proteínas y actúa como antioxidante y cicatrizante. También favorece la absorción de hierro procedente de los alimentos de origen vegetal.

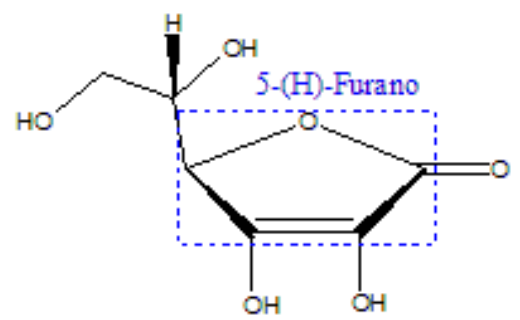


Figura 13. Vitamina E.

\section{Vitamina E}

La vitamina E tiene el heterociclo denominado cromano, el cual es un biciclo que tiene como heteroátomo un oxígeno (figura 13). Esta vitamina interviene en la formación de ADN y ARN; participa en la formación de los glóbulos rojos, músculos y otros tejidos; también actúa en los procesos de cicatrización. Participa en las reacciones de destrucción de los llamados radicales libres, los cuales son indeseables debido a que en el mejor de los casos causan envejecimiento pero también están directamente relacionados con graves enfermedades.

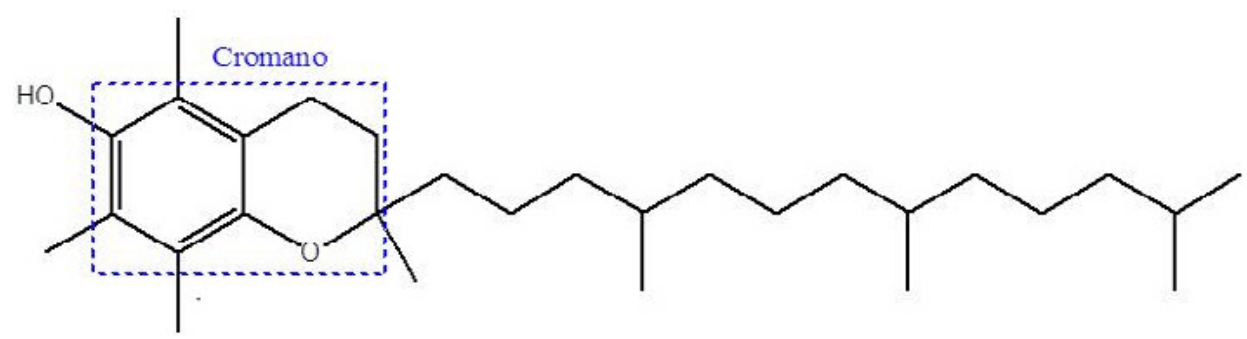

\section{Hormonas}

Las hormonas se comportan como mensajeros químicos en el cuerpo, por lo cual su aumento o disminución provoca determinadas emociones como se describe a continuación.

\section{Hormona oxitocina}

La oxitocina es una hormona que está formada por diferentes aminoácidos y uno de ellos es la prolina, el cual, como ya se mencionó anteriormente, posee el heterociclo pirrolidina (figura 14). La oxitocina está implicada en todos los procesos afectivos, en los cuales se ha visto que ayuda a forjar lazos permanentes entre parejas, además afianza el vínculo entre una madre y su bebé.

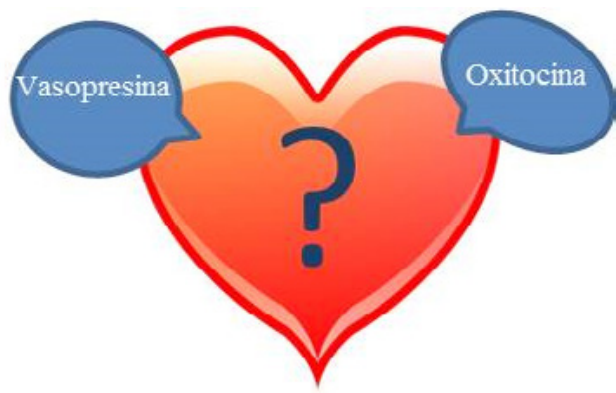
Se produce tanto en un parto como en una relación de pareja, ya que se libera por un simple abrazo o caricia. Es por este motivo que la conocemos como "la hormona del amor", ya que su presencia fomenta los vínculos interpersonales y provoca un fuerte sentimiento de cariño hacia amigos, compañeros, familiares, etcétera (Behnia et al., 2014; Magon y Kalra, 2011). 
Figura 14. Oxitocina.

Figura 15. Vasopresina.

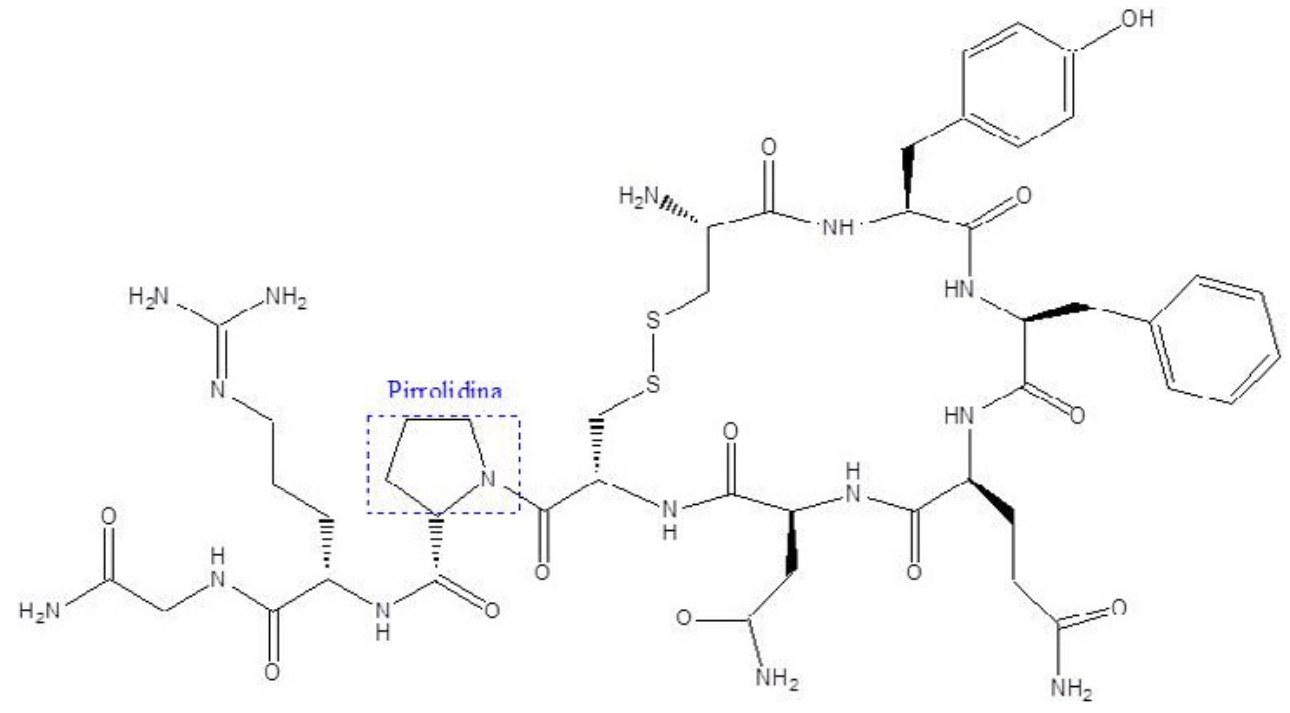

\section{Hormona vasopresina}

Por otra parte, y no menos importante como estructura química, está la vasopresina, implicada también en el proceso afectivo. Al enamorarse de otra persona esta sustancia química se incrementa en nuestro organismo y hace que el vínculo se prolongue durante muchos años, por lo cual se le conoce como "sustancia química de la monogamia" (Donaldson y Young, 2008). Al igual que la oxitocina, está formada por una serie de aminoácidos y uno de ellos es la prolina, que contiene el heterociclo pirrolidina (figura 15).

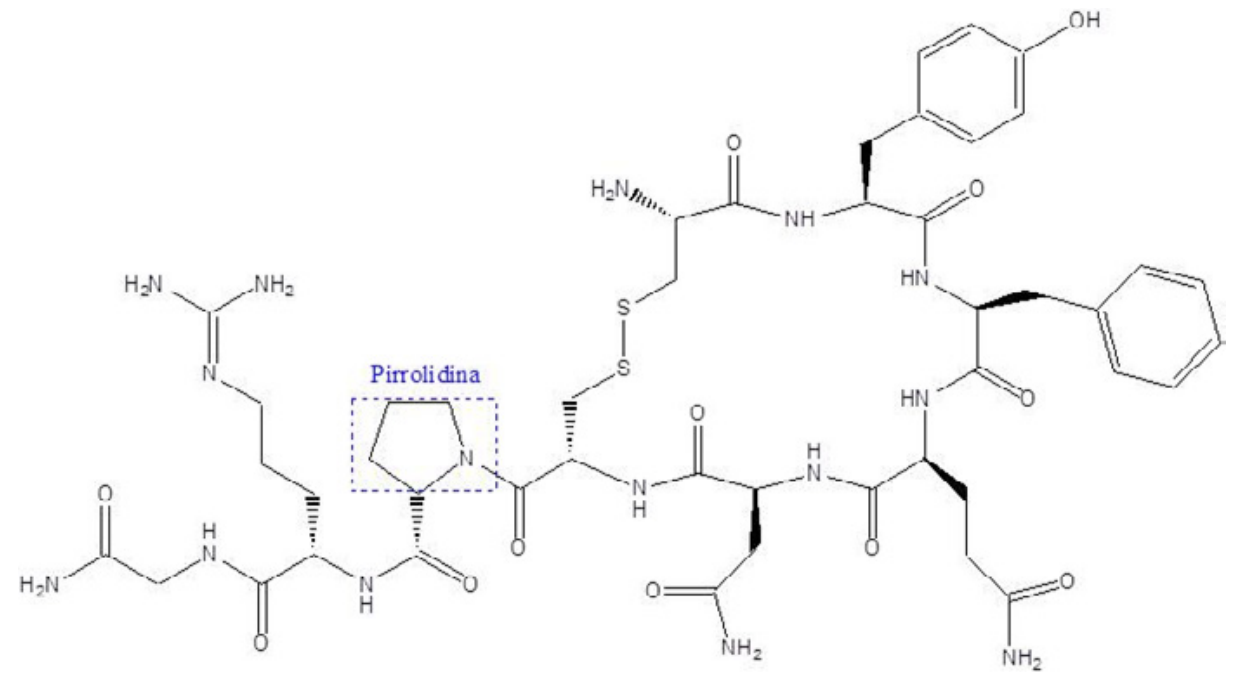


Figura 16. Serotonina.
Figura 17. Melatonina.

\section{Hormona serotonina}

La serotonina es una estructura química muy pequeña que tiene como base principal un heterociclo llamado indol. Es conocida como la "hormona del placer" además de ser la "hormona del humor" (figura 16). Desde un punto de vista más amplio, la serotonina nos provoca felicidad. Se ha demostrado que los niveles de serotonina están directamente relacionados con las estaciones del año (Praschak et al., 2008). Además, está implicada en los cambios de humor durante el ciclo premenstrual, ya que se disminuye los niveles de serotonina en el organismo femenino. Cuando ésta llega a niveles muy bajos, pueden suceder episodios de irritabilidad, ansiedad y hasta depresión en mujeres con síndrome premenstrual (spm) (León-García, 2015). Además, regula numerosos procesos físicos y mentales como el apetito, los niveles de ansiedad, el sueño, el comportamiento sexual y conductas adictivas,

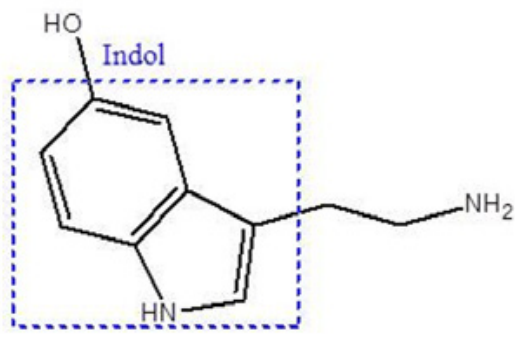
entre otros.

\section{Hormona melatonina}

La melatonina también tiene un anillo heterocíclico llamado indol (figura 17). Al igual que las otras estructuras químicas de nuestro organismo, tiene diferentes funciones, un ejemplo es la de regular nuestro reloj biológico (Cardinali et al., 2012). La melatonina aumenta por la noche y nos provoca sueño; sin embargo, por la mañana disminuye y nos hace despertar. Por otra parte, estimula la secreción de la hormona del crecimiento; por eso se suele decir que crecemos al dormir. Como es de esperar, la cantidad de melatonina es más alta en los niños y en la vejez disminuye considerablemente. Por lo anterior, los niños tienen la necesidad de dormir mucho más que un adulto.

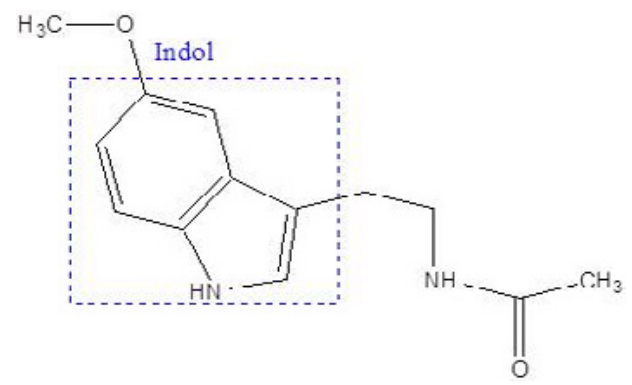

\section{Neurotransmisor endorfina}

La endorfina está compuesta por dos heterociclos, el furano y la piperidina, fusionados a través del hexahidrofenantreno (figura 18). Nuestro organismo 
Figura 18.

Endorfina.

tiene la capacidad de disminuir el dolor a través de los analgésicos naturales del cuerpo llamados endorfinas. Se trata de pequeñas proteínas de las cuales existen alrededor de veinte tipos diferentes. Estas moléculas se producen cuando realizamos ejercicio intenso (Gbenga y David, 2015), ya que nuestro cuerpo siente inflamación y comienza a producir endorfinas para compensar el dolor, provocando una sensación de bienestar. También se producen en el cuerpo por tacto con personas queridas, ya que los receptores debajo de la piel registran esta sensación y la transmiten a las terminaciones nerviosas, provocando una corriente eléctrica que llega hasta el cerebro, lo cual permite liberar las endorfinas, provocando placer.

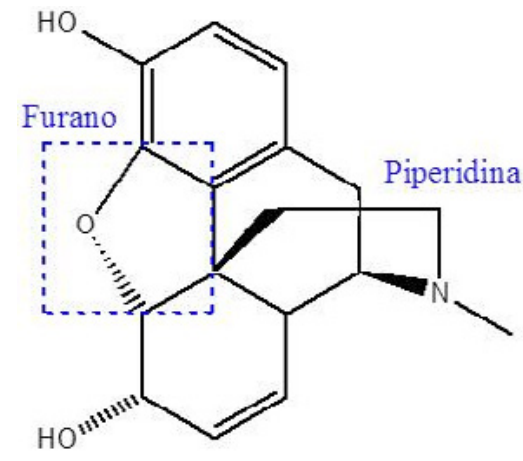

\section{Hemoglobina}

Una estructura fundamental en los seres humanos es la hemoglobina, todos hemos escuchado hablar de ella. Se trata de una proteína conjugada bastante compleja, pero una de sus partes principales es el grupo hemo, el cual está constituido por cuatro heterociclos llamados pirrol y en el centro tiene un átomo de hierro

Figura 19. Hemoglobina. (figura 19), lo cual le confiere un sabor característico (Vera, 2010). Esta proteína está contenida en los glóbulos rojos o eritrocitos y su función principal es el transporte de oxígeno y dióxido de carbono en el proceso de respiración celular.

\section{Conclusión}

Los heterociclos, al igual que cualquier otro compuesto químico, forman parte de algunas estructuras imprescindibles en nuestro organismo, por lo cual conocer su configuración

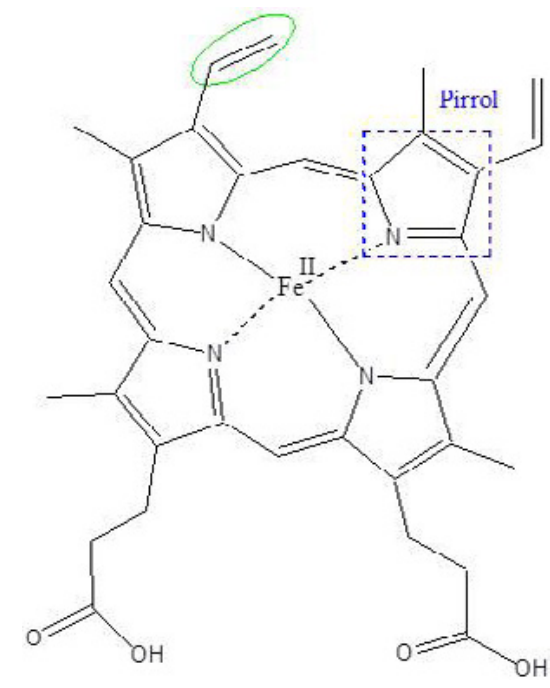


nos permite identificarlos de manera general y descubrir que, aunque son pequeños anillos, tienen extraordinarias funciones. Debemos recordar que todos y cada uno de los procesos que se llevan a cabo en cualquier organismo vivo forman parte de una serie de reacciones químicas en las cuales los heterociclos pueden estar presentes.

\section{Referencias}

* Behnia, B., Heinrichs, M., Bergmann, W., Jung, S., Germann, J., Schedlowski, M. y Kruger, T. H. (2014). Differential effects of intranasal oxytocin on sexual experiences and partner interactions in couples. Hormones and behavior, 65, 308-318. Dol: https://doi.org/10.1016/j.yhbeh.2014.01.009.

- Cardinali, D. P., Srinivasan, V., Brzezinski, A. y Brown, G. M. (2012). Melatonin and its analogs in insomnia and depression. Journal of pineal research, 52, 365-375. Dol: https://doi.org/10.1111/j.1600-079X.2011.00962.x.

* Donaldson, Z. R. y Young, L. J. (2008). Oxytocin, vasopressin, and the neurogenetics of sociality. Science, 322, 900-904. Dol: https://doi.org/10.1126/science.1158668.

* Gbenga, J. B. yDavid, B. T. (2015). The increasing relevance of physical activity and exercise to individual mental health. Afro asian journal of science and technology, 2, 192-198. Recuperado de: http://aajst.com/wp-content/uploads/2015/06/Jaiyesimi-Boluwaji-Gbenga-et-al-Afro-Asian-J-SciTech201521192-198.pdf.

León-García, M. (2015). Serotonina, ciclo menstrual y síndrome premenstrual. Medicina naturista, 9, 103-108.

* Magon, N. y Kalra, S. (2011). The orgasmic history of oxytocin: Love, lust, and labor. Indian journal of endocrinology and metabolism, 15, 156-161. Dol: https://doi. org/10.4103/2230-8210.84851.

* Mathews, C. K., Van Holde, K. E. y Ahern, K. G. (2002). Bioquímica, (3ª ed.), Madrid, España: Pearson Educación.

Murray, R.K., Bender, D.A., Botham, K.M., Kennelly, P.J., Rodwell, V. W. y Weil, P.A. (Eds.) (2010). Harper. Bioquímica ilustrada, (28ª ed.), México, México: Mac Graw-Hill.

* Pardo, A. V. P. (2004). La importancia de las vitaminas en la nutrición de personas que realizan actividad físico deportiva. Revista internacional de medicina y ciencias de la actividad física y el deporte, 4, 233-242. Recuperado de: http://cdeporte. rediris.es/revista/,revista16/artvitamina.pdf.

* Praschak-Rieder, N., Willeit, M., Wilson, A. A., Houle, S. y Meyer J. H. (2008). Seasonal variation in human brain serotonin transporter binding. Archives of general psychiatry, 65, 1072-1078. Dol: https://doi.org/10.1001/archpsyc.65.9.1072.

* Vera, L. F. (2010). La Hemoglobina: una molécula prodigiosa. Real academia de ciencias exactas, físicas y naturales, 104, 213-232. Recuperado de: http://www.rac.es/ ficheros/doc/00906.pdf. 


\section{Cómo citar este artículo}

* Hernández Romero, D., García Barradas, O, Colorado Peralta, R. y Sánchez Pavón, E. (2018). Heterociclos: pequeñas y maravillosas estructuras en el organismo humano. Revista Digital Universitaria (RDU). Vol. 19, núm. 4 julio-agosto. DOI: http://doi. org/10.22201/codeic.16076079e.2018.v19n4.a3. 